\title{
Wound Healing on the Dorsal Hands: An Intrapatient Comparison of Primary Closure, Purse-String Closure, and Secondary Intention
}

\author{
Jayson Miedema, MD; Ling-Lun Bob Hsia, MD; Rajat Varma, MD
}

Nonmelanoma skin cancers are common on the dorsal hands where reserve tissue is limited. We highlight the case of an elderly man who had 3 nonmelanoma skin cancers on the left hand that were treated on the same day and left similar wounds. The wounds were repaired by primary closure, secondary intention, and purse-string circumferential closure. All wounds healed with excellent and essentially equivalent cosmetic results. For small shallow wounds on the dorsal hands, dermatologic surgeons should have confidence that secondary intention healing likely will lead to acceptable cosmetic and functional results.

Cutis. 2021; 107:318-319.

\section{Practice Gap}

Many cutaneous surgery wounds can be closed primarily; however, in certain cases, other repair options might be appropriate and should be evaluated on a case-by-case basis with input from the patient. Defects on the dorsal aspect of the hands - where nonmelanoma skin cancer is common and reserve tissue is limited-often heal by secondary intention with good cosmetic and functional results. Patients often express a desire to reduce the time spent in the surgical suite and restrictions on postoperative activity, making secondary intention healing more appealing. An additional advantage is obviation of the need to remove additional tissue in the form of Burow triangles, which would lead to a longer wound. The major disadvantage of secondary intention healing is longer time to wound maturity; we often minimize this disadvantage with purse-string closure to decrease the size of the wound defect, which can be done quickly and without removing additional tissue.

\section{The Technique}

An elderly man had 3 nonmelanoma skin cancers-all on the dorsal aspect of the left hand-that were treated on the same day, leaving 3 similar wound defects after Mohs micrographic surgery. The wound defects (distal to proximal) measured $12 \mathrm{~mm}, 12 \mathrm{~mm}$, and $10 \mathrm{~mm}$ in diameter (Figure 1) and were repaired by primary closure, secondary intention, and purse-string circumferential closure, respectively. Purse-string closure ${ }^{1}$ was performed with a 4-0 polyglactin 901 suture and left to heal without external sutures (Figure 2). Figure 3 shows the 3 types of repairs immediately following closure. All wounds healed with excellent and essentially equivalent cosmetic results, with excellent patient satisfaction at 6 -month follow-up (Figure 4).

\section{Practical Implications}

Our case illustrates different modalities of wound repair during precisely the same time frame and essentially on the same location. Skin of the dorsal hand often is tight; depending on the size of the defect, large primary closure can be tedious to perform, can lead to increased wound

Drs. Miedema and Varma are from the Department of Dermatology, University of North Carolina at Chapel Hill. Dr. Miedema also is from the Department of Pathology. Dr. Hsia is from the Department of Dermatology, Medical University of South Carolina, Charleston.

The authors report no conflict of interest.

Correspondence: Jayson Miedema, MD, 410 Market St, Ste 400, Chapel Hill, NC 27516 (jayson_miedema@med.unc.edu). doi:10.12788/cutis.0265 


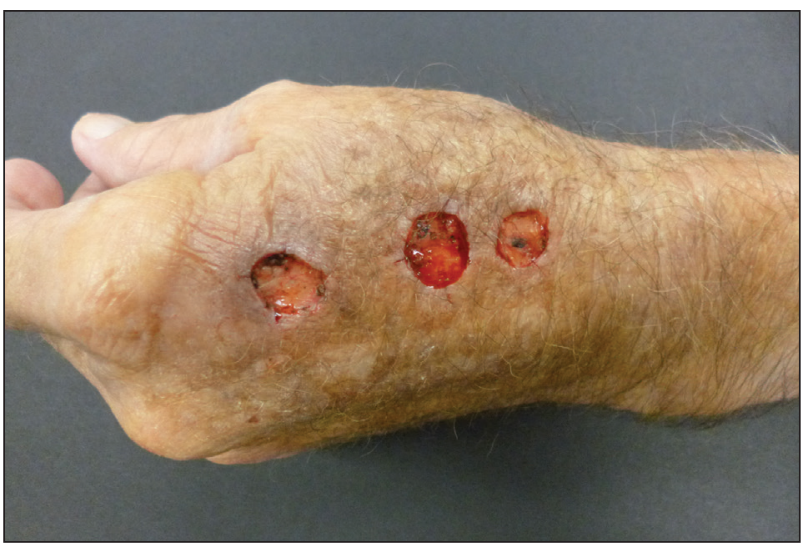

FIGURE 1. Wounds prior to closure.

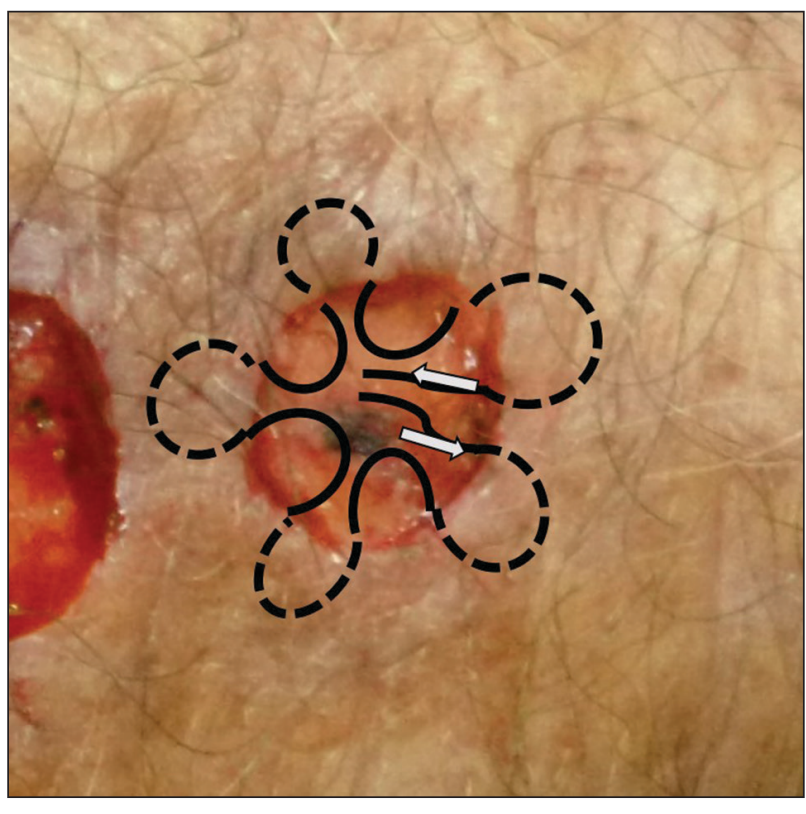

FIGURE 2. A dermal purse-string closure suture pathway was used for one of the wounds to reduce wound size.

tension and risk of dehiscence, and can be uncomfortable for the patient during healing. However, primary closure typically will lead to faster healing.

Secondary intention healing and purse-string closure require less surgery and therefore cost less; these modalities yield similar cosmesis and satisfaction. In the appropriate context, secondary intention has been highlighted as a suitable alternative to primary closure ${ }^{2-4}$; in our experience (and that of others ${ }^{5}$ ), patient satisfaction is not diminished with healing by secondary intention. Purse-string closure also can minimize wound size and healing time.

For small shallow wounds on the dorsal hand, dermatologic surgeons should have confidence that secondary

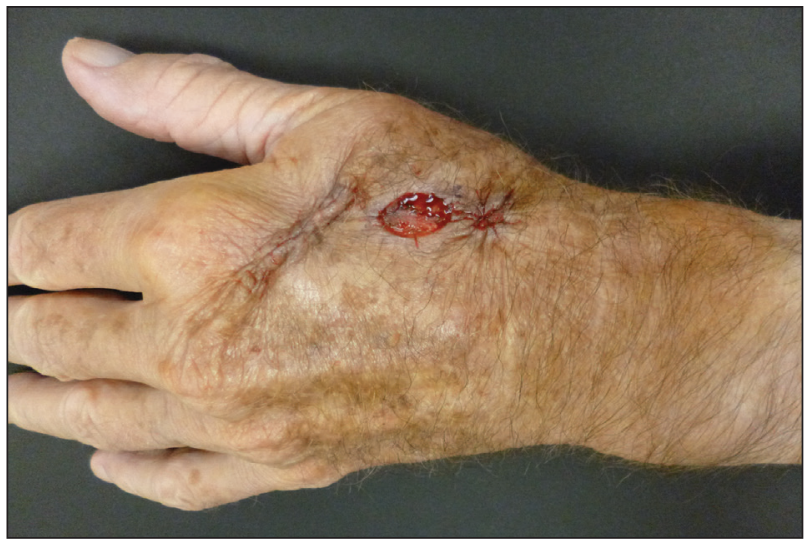

FIGURE 3. Wounds immediately following repair (distal to proximal: linear closure, secondary intention, purse-string closure).

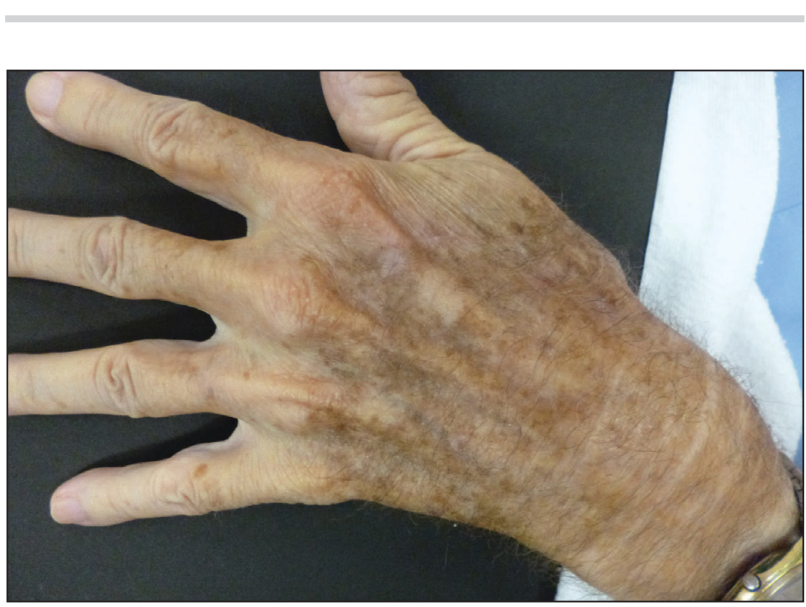

FIGURE 4. At 6-month follow-up, there was essentially no difference in the appearance of the 3 wounds.

intention healing, with or without wound reduction using purse-string repair, likely will lead to acceptable cosmetic and functional results. Of course, repair should be tailored to the circumstances and wishes of the individual patient.

\section{REFERENCES}

1. Peled IJ, Zagher U, Wexler MR. Purse-string suture for reduction and closure of skin defects. Ann Plast Surg. 1985;14:465-469. doi:10.1097/00000637-198505000-00012

2. Zitelli JA. Secondary intention healing: an alternative to surgical repair. Clin Dermatol. 1984;2:92-106. doi:10.1016/0738-081x(84)90031-2

3. Fazio MJ, Zitelli JA. Principles of reconstruction following excision of nonmelanoma skin cancer. Clin Dermatol. 1995;13:601-616. doi:10.1016/0738-081x(95)00099-2

4. Bosley R, Leithauser L, Turner M, et al. The efficacy of second-intention healing in the management of defects on the dorsal surface of the hands and fingers after Mohs micrographic surgery. Dermatol Surg. 2012;38:647-653. doi:10.1111/j.1524-4725.2011.02258.x

5. Stebbins WG, Gusev J, Higgins HW 2nd, et al. Evaluation of patient satisfaction with second intention healing versus primary surgical closure. J Am Acad Dermatol. 2015;73:865-867.e1. doi:10.1016/j. jaad.2015.07.019 